\title{
Neue Therapieempfehlungen für STD Nützliches für den dermatologischen Alltag
}

\section{Sexually Transmitted Diseases: New Treatment Guidelines Valuable Recommendations for Daily Practice}

\author{
Autor \\ J. Weiß
}

\section{Bibliografie}

Dol $10.1055 / \mathrm{s}-2007-995758$

Akt Dermatol 2008; 34:

157-162 @ Georg Thieme

Verlag KG Stuttgart · New York ISSN 0340-2541

Korrespondenzadresse

Prof. Dr. Jürgen Weiß

Dermatologische Gemeinschaftspraxis Prof. Dr. J. Weiß und Dr. M. Kaspari

Osterstr. 24

30159 Hannover

j_weiss@gmx.de

\section{Zusammenfassung}

In den letzten Jahren haben nationale und internationale Fachgesellschaften und Gesundheitsorganisationen in immer kürzeren Abständen neue Therapieleitlinien zur Behandlung von sexuell übertragbaren Erkrankungen (STD) veröffentlicht. Diese wurden erforderlich, weil weltweit ein deutlicher Anstieg der Inzidenz beobachtet wird, im Zuge der Globalisierung rasche Resis-

Die Behandlung von Geschlechtskrankheiten zählt (noch?) zur Kernkompetenz der Dermatologen. Der Anteil der Patienten mit sexuell übertragbaren Erkrankungen ist im Vergleich zu dem von Dermatosen sicherlich von untergeordneter Bedeutung. Der deutliche Rückgang der Erkrankungszahlen sexuell übertragbarer Krankheiten in den letzten Jahrzehnten hat aber zu einem Rückgang der Erfahrung damit geführt. Diese für unser Fach problematische Entwicklung wird konterkariert durch einen massiven Inzidenzanstieg der Krankheiten in den letzten 10 Jahren. Es ist zu erwarten, dass die Infektionszahlen mit dem Zusammenwachsen Europas weiter ansteigen, zumal in Osteuropa die Inzidenzen um ein Mehrfaches höher sind als in Deutschland. Im Zeitalter der Leitlinienmedizin ist der Hautarzt sicherlich gut beraten, entsprechende Therapieempfehlungen von Fachgesellschaften zu berücksichtigen. In den letzten Jahren haben nationale und internationale Fachgesellschaften und Gesundheitsorganisationen eine Flut neuer Therapieleitlinien veröffentlicht und aktualisiert, die mikrobiologische und pharmakologische Fortschritte aufnehmen. Hervorzuheben sind die Empfehlungen der Centers for Disease Control and Prevention CDC (Sexually Transmitted Diseases Treatment Guidelines 2006 [1,2]), der IUSTI (International Union against Sexually Transmitted Infections - European STD Guidelines 2001 tenzausbreitungen auftreten und Fortschritte in der Pharmakotherapie aufgenommen werden. Die Unterschiede in den Empfehlungen der nationalen und internationalen Therapieleitlinien beruhen eher auf dem Zeitpunkt der Erstellung als auf regionalen Besonderheiten. Der folgende kurze Abriss stellt aktuelle Aspekte in der Epidemiologie und Therapie dar, welche im täglichen Umgang mit betroffenen Patienten relevant sind.

[3] und Draft European Guideline on Syphilis 2007 [4]) sowie der Diagnostik- und Therapieleitlinie der Syphilis der deutschen STD Gesellschaft (DSTDG) von 2005 [5]. Für 2008 haben sowohl IUSTI/WHO als auch die DSTDG Revisionen in ihren Therapieleitlinien angekündigt.

Im Folgenden sollen die jüngsten Therapieempfehlungen der CDC und der IUSTI unter dem Aspekt der Bedürfnisse des praktizierenden Hautarztes vorgestellt werden. Bei speziellen Fragestellungen und therapeutischen Problemen sollte immer aber auf die Originalpublikationen und vor allem deren aktuelle Updates zurückgegriffen werden. Im Literaturverzeichnis finden sich deshalb bevorzugt Quellen mit elektronischer Publikation und deren Web-Adressen.

\section{LUES}

$\nabla$

Das Zusammenwachsen Europas hat eine Renaissance der bekanntesten Geschlechtskrankheit zur Folge. In Deutschland wurden 20063147 neue Fälle der Syphilis vom Robert Koch Institut erfasst [6]. Dies stellt nahezu eine Verdreifachung der Inzidenz im Vergleich zu 2000 dar. Diese liegt bei etwa 3 pro 100000 Einwohnern und damit um den Faktor 5-10 unter den Zahlen von Osteuropa [7]. Der Rückgang der Inzidenz in den USA hatte dazu geführt, dass vor gut 10 Jah- 
Tab. 1 Therapieempfehlungen für Lues

\begin{tabular}{|c|c|c|}
\hline Deutsche STD-Gesellschaft 2005 & IUSTI Guidelines 2007 & CDC Guidelines 2006 \\
\hline $\begin{array}{l}\text { Benzathinpenicillin G 2,4MU einmalig } \\
\text { Procain-Penicillin* 1,2MU/Tag } 14 \text { Tage }\end{array}$ & $\begin{array}{l}\text { Frühformen } \\
\text { (Lues I, Lues II bis } 12 \text { Monate nach Infektion) } \\
\text { Benzathinpenicillin G 2,4MU einmalig } \\
\text { Procain-Penicillin* } 0,6 \mathrm{MU} / \mathrm{Tag} \\
10-14 \text { Tage }\end{array}$ & Benzathinpenicillin G 2,4MU einmalig \\
\hline $\begin{array}{l}\text { Doxycyclin } 2 \times 100 \mathrm{mg} / \mathrm{d} \text { Erythromycin** } \\
4 \times 500 \mathrm{mg} / \mathrm{d} \\
\text { jeweils } 14 \text { Tage } \\
\text { oder } \\
\text { Ceftriaxon } 1 \mathrm{~g} / \mathrm{d} \text { iv } 10 \text { Tage } \\
\text { Tetrazyklin } 4 \times 500 \mathrm{mg} / \mathrm{d} 14 \text { Tage }\end{array}$ & $\begin{array}{l}\text { Bei Penicillinallergie } \\
\text { Doxycyclin } 2 \times 100 \mathrm{mg} / \text { Tag Tetrazyklin } 4 \times 500 \mathrm{mg} / \text { Tag } \\
\text { Erythromycin** } 4 \times 500 \mathrm{mg} / \mathrm{Tag} \\
\text { jeweils } 14 \text { Tage } \\
\text { Azithromycin** } 2 \mathrm{~g} \text { (Einzeldosis) }\end{array}$ & $\begin{array}{l}\text { Doxycyclin } 2 \times 100 \mathrm{mg} / \text { Tag oder } \\
\text { Tetrazyklin } 4 \times 500 \mathrm{mg} / \text { Tag } \\
\text { jeweils } 14 \text { Tage } \\
\text { Ceftriaxone } 1 \mathrm{~g} / \text { die } 8-10 \text { Tage }\end{array}$ \\
\hline $\begin{array}{l}\text { Benzathinpenicillin G 2,4MU } \\
3 \text { Injektionen Tag 1,8,15 } \\
\text { oder } \\
\text { Procain-Penicillin* 1,2MU/Tag } 21 \text { Tage }\end{array}$ & $\begin{array}{l}\text { Infektion älter als } 12 \text { Monate } \\
\text { Benzathinpenicillin } \mathrm{G} 2,4 \mathrm{MU} \\
3 \text { Injektionen Tag } 1,8,15 \\
\text { oder } \\
\text { Procain-Penicillin* } 0,6 \mathrm{MU} / \text { Tag } 17-21 \text { Tage }\end{array}$ & $\begin{array}{l}\text { Benzathinpenicillin G 2,4MU } \\
3 \text { Injektionen Tag 1, 8, } 15\end{array}$ \\
\hline $\begin{array}{l}\text { Doxycyclin } 2 \times 100 \mathrm{mg} / \text { Tag } \\
28 \text { Tage oder } \\
\text { Erythromycin }{ }^{* *} 2 \mathrm{~g} / \mathrm{d} \text { iv } \\
21 \text { Tage } \\
\text { Ceftriaxon } 1 \mathrm{~g} / \mathrm{d} \text { iv } 14 \text { Tage }\end{array}$ & $\begin{array}{l}\text { Bei Penicillinallergie } \\
\text { Doxycyclin } 2 \times 100 \mathrm{mg} / \mathrm{Tag} 21-28 \text { Tage oder } \\
\text { Tetrazyklin } 4 \times 500 \mathrm{mg} / \mathrm{Tag} \\
\text { Erythromycin** } 4 \times 500 \mathrm{mg} / \mathrm{Tag} \\
\text { jeweils } 28 \text { Tage }\end{array}$ & $\begin{array}{l}\text { Doxycyclin } 2 \times 100 \mathrm{mg} / \text { Tag } \\
\text { Tetrazyklin } 4 \times 500 \mathrm{mg} / \text { Tag } \\
\text { jeweils } 28 \text { Tage }\end{array}$ \\
\hline
\end{tabular}

ren Programme gestartet wurden, diese Infektion auszurotten. Wie in Westeuropa steigen auch hier die Inzidenzzahlen wieder massiv an. 2006 wurden etwa 10000 neue Fälle beobachtet, wovon mehr als 300 kongenitale Erkrankungen waren. [2]

Die Luesbehandlung kann ambulant erfolgen. Die praktikable Therapie für die Praxis ist nach dieser Übersicht die Injektion von Benzathin-Penizillin (s. Tab. 1). Sowohl Pendysin ${ }^{\circledR} 1,2 \mathrm{MU}$ und jetzt auch wieder Tardocillin ${ }^{\circledR} 1,2 \mathrm{MU}$ sind für diese Indikation zugelassen. Es ist empfehlenswert, die im-Injektion beider Ampullen getrennt in jede Glutäusseite zu verabreichen. Die Injektion kann Beschwerden für einige Tage verursachen. Bei florider Syphilis tritt in den ersten 24 Stunden nach der Erstinjektion eine Jarisch-Herxheimer Reaktion auf. Diese läßt sich durch die Gabe von Prednisolon (50-100 mg) und Antipyretika gut beherrschen.

Bei Bestehen einer Penicillinallergie kann auf eine Tetrazyklinbehandlung ausgewichen werden. Nach den europäischen/deutschen Leitlinien kann auch eine Erythromycinbehandlung erfolgen. Hierbei ist aber zu berücksichtigen, dass inzwischen auch in Europa resistente Treponemenstämme bekannt sind. Bei diesen Alternativtherapien ist unbedingt auf eine serologische Kontrolle der Sanierung zu achten.

Die Lues ist nach Infektionsschutzgesetz (IfCS) meldepflichtig. Eine Meldung an das Robert Koch Institut (RKI) erfolgt anonym durch den Laborarzt, sobald er eine reaktive Luesserologie nachweist. Der behandelnde Hautarzt hat vom Patienten epidemiologische Daten zu erheben, die ebenfalls an das RKI in Berlin weitergeleitet werden.

In Deutschland zeigen epidemiologische Daten eine signifikante Rate einer Koinfektion mit HIV. Aus diesem Grund sollten alle Luespatienten auch (eventuell wiederholt) auf eine Retrovirusinfektion getestet werden. Die Luestherapie bei HIV-Patienten folgt dem genannten Schema. Einzelne Therapeuten empfehlen auch bei der Frühsyphilis eine dreimalige Penicillininjektion.
Bei späten Luesformen sollte dann aber eine Neurolues durch Punktion ausgeschlossen sein.

Die Behandlung der Lues in der Schwangerschaft unterscheidet sich ebenfalls nicht von der Normaltherapie. Da Doxycyclin in der Schwangerschaft kontraindiziert und Erythromycin beim Fetus nicht sicher wirksam ist, sollte bei Bestehen einer Penicillinallergie eine spezifische Hyposensibilisierung und anschließende Therapie mit Penicillin durchgeführt werden. Bei der Therapie ist zu berücksichtigen, dass eine Jarisch-Herxheimer-Reaktion in der Spätschwangerschaft Wehen induzieren kann.

Nach erfolgter Luestherapie sind Folgeuntersuchungen unbedingt erforderlich, um Therapieversager auszuschließen. Die CDC gibt hierzu eine Häufigkeit bis $15 \%$ an. Die empfohlenen klinischen und serologischen Kontrollen sollten nach 6 und 12 Monaten stattfinden. Die erfolgreiche Therapie zeigt sich dabei in einer deutlichen Reduktion (mindestens Faktor 4) oder Verschwinden der VDRL oder RPR Antikörper. Um eine Vergleichbarkeit der Titer zu gewährleisten, sollte darauf geachtet werden, dass die Laboruntersuchungen im gleichen Labor und mit der gleichen Methode durchgeführt werden. Titerschwankungen des TPHA oder des FTA haben keine diagnostische Wertigkeit. Sollte die angeführte Titerreduktion von VDRL oder RPR nicht eintreten, ist von einem Therapieversager (oder einer Reinfektion) auszugehen. In diesen Fällen hält die CDC eine erneute Therapie einschließlich einer Lumbalpunktion für erforderlich. Die CDC geht davon aus, dass bei HIV-Patienten die Rate der Therapieversager sowie die Inzidenz einer Neurolues (minimal) erhöht sein könnte. Aus diesem Grund ist eine Infektionssanierung durch serologischen Verlaufskontrollen 3, 6, 9, 12 und 24 Monaten nach Therapie nachzuweisen. Die Wertigkeit einer Lumbalpunktion 6 Monate nach Therapie einer Frühsyphilis ist nicht geklärt.

Die Lues latens seropositiva wird bei Fehlen zeitlich eindeutig einzuordnender primärer oder sekundärer Luesmanifestationen 
Tab. 2 Therapieempfehlungen für Gonorrhoe

\section{Deutsche STD-Gesellschaft 2001}

Spectinomycin $2 \mathrm{~g}$ i. m. einmalig

Ceftriaxon $250 \mathrm{mg}$ i. m. einmalig

\section{Alternativ}

Cefixim $400 \mathrm{mg}$ po einmalig

Ciprofloxacin* $500 \mathrm{mg}$ po einmalig

Ofloxacin* $400 \mathrm{mg}$ po einmalig

Azithromycin $1 \mathrm{~g}$ po einmalig
IUSTI Guidelines 2001

Für Urethra, Cervix und Rectum

Ceftriaxon $125 \mathrm{mg}$ i.m. einmalig Ciprofloxacin* $500 \mathrm{mg}$ po einmalig Ofloxazin* $400 \mathrm{mg}$ po einmalig Cefixim $400 \mathrm{mg}$ po einmalig Spectinomycin $2 \mathrm{~g}$ i. m. einmalig

\section{CDC Guidelines 2006}

Cefixim $400 \mathrm{mg}$ po einmalig Ceftriaxon $125 \mathrm{mg}$ i. m. einmalig Plus Chlamydientherapie, falls Infektion nicht ausgeschlossen

\section{Alternativ}

Spectinomycin $2 \mathrm{~g}$ i. m. einmalig

Oder anderes Cephalosporin

Ceftriaxon $125 \mathrm{mg}$ i. m. einmalig

\section{Für Pharynx}

Ceftriaxon $125 \mathrm{mg}$ i.m. einmalig

Ciprofloxacin* $500 \mathrm{mg}$ po einmalig

Ofloxazin* $400 \mathrm{mg}$ po einmalig
Plus Chlamydientherapie, falls Infektion nicht ausgeschlossen

Cave:

* Gyrasehemmer sollten wegen häufiger Resistenz nicht mehr eingesetzt werden.

als Spätsyphilis eingeordnet und wie eine solche behandelt. Die Diagnosestellung wird hier meist zufällig erfolgen. Vor Therapieeinleitung ist eine subtile dermatologische Suche nach (narbig abgeheilten) Primäraffekten und der Ausschluss von Gummen und anderen tertiären Luesmanifestationen erforderlich. Auch sollte eine augenärztliche Konsiliaruntersuchung (Uveitis), eine neurologische Konsiliaruntersuchung (Neurolues), sowie eine Röntgenaufnahme des Thorax (Aneurysma der Aorta ascendens) durchgeführt werden. Die CDC hält eine Lumbalpunktion für erforderlich, wenn sich bei diesen Untersuchungen Auffälligkeiten ergeben oder der Patient zusätzlich eine HIV Infektion aufweist.

\section{Gonorrhoe}

$\nabla$

Der Tripper ist nicht mehr meldepflichtig. Genaue Inzidenzzahlen für Deutschland liegen deshalb nicht mehr vor. Die Rate der Neuerkrankungen ist in Westeuropa in den letzten 25 Jahren drastisch zurückgegangen, die WHO gibt jetzt eine Inzidenz von etwa 5 Neuerkankungen/100 000 Einwohner und Jahr an [5]. Bei Nachweis eines Trippers sollte eine Doppelinfektion mit HIV oder Lues ausgeschlossen werden.

Die unkomplizierte genitale oder rektale Gonorrhoe verursacht Beschwerden. Der färberische Direktnachweis von Gonokokken im Grampräparat wird im positiven Falle für eine Diagnosebestätigung als ausreichend erachtet. Der kulturelle Nachweis von Gonokokken erfordert die Verwendung von speziellen Kulturmedien und Transportbedingungen. Diese sind meist nicht vorhanden oder erfüllbar. Der labormedizinische Befund Gonokokken negativ bei Verwendung der üblichen bakteriologischen Abstrichröhrchen muss deshalb streng hinterfragt werden. Als Alternative bietet sich der Gonokokken-Nachweis mittels PCR an, der jedoch keine Resistenzbestimmung erlaubt.

In der Praxis bietet sich, auch unter Kostengesichtspunkten, die einmalige Gabe von 400 mg Cefixim (1 Tablette) po als Standardtherapie der unkomplizierten genitalen oder anorektalen Gonorrhoe an (s. Tab. 2), gefolgt von einer anschließenden Doxycyclingabe (200 mg/die über 7 Tage). In Amerika wird eine solche routinemäßige Dualtherapie gegen Gonokokken und Chlamydien damit begründet, dass Doppelinfektionen in 10-30\% der Fälle bestehen. Die neueren Inzidenzzahlen legen ähnliche Ver- hältnisse für Chlamydieninfektionen auch in Deutschland nahe [8].

In den deutschen und europäischen Therapieempfehlungen finden sich noch Gyrasehemmer. In Osteuropa sind aktuell die Gonokokkenstämme nahezu komplett gegen diese Antibiotikagruppe resistent. Gyrasehemmer sollten deshalb nur noch eingesetzt werden, wenn eine Resistenz ausgeschlossen ist.

Im Gegensatz zur Syphilis werden bei der Gonorrhoe keine Nachuntersuchungen mehr für erforderlich gehalten, wenn der Patient durch die Therapie beschwerdefrei wird. Bei persistierenden Beschwerden ist eine umfangreiche Diagnostik erforderlich, die klären muss, ob eine bakterielle Resistenz gegen das Primärtherapeutikum, eine Gonorrhoe-Reinfektion oder das klinische Hervortreten einer Doppelinfektion besteht. Hierbei ist zu berücksichtigen, dass der PCR-Nachweis von Gonokokken in diesen Fällen zu falsch-positiven Ergebnissen führen kann (Persistenz avitaler Keime).

Die nicht-gonorrhoische Urethritis kann wie eine Chlamydieninfektion mit einer einmaligen Gabe von $1 \mathrm{~g}$ Azithromycin oder $200 \mathrm{mg} /$ die Doxycyclin über 7 Tage behandelt werden. Für Therapieversager oder Rezidive wird die einmalige Gabe von $2 \mathrm{~g}$ Metronidazol plus einer 7-tägigen Erythromycinbehandlung $(4 \times 500 \mathrm{mg} / \mathrm{die})$ empfohlen.

\section{Chlamydieninfektionen \\ $\nabla$}

Chlamydieninfektionen sind die häufigsten Auslöser der nichtgonorrhoischen Urethritis oder der mucopurulenten Zervizitis. Genaue Inzidenzzahlen für Deutschland liegen nicht vor, da die Infektion nicht meldepflichtig ist. 2005 wurden im deutschen Ärzteblatt Prävalenzzahlen von Chlamydieninfektionen bei Heranwachsenden veröffentlicht [8]. Man stieß auf eine unerkannte Epidemie: 10\% der sexuell aktiven 17-20-jährigen Frauen weisen eine (asymptomatische) Infektion auf. Ähnliche drastische Inzidenz-/Prävalenzanstiege werden auch für andere westeuropäische Länder berichtet. In den USA scheint die Prävalenz, zumindest in bestimmten Gebieten, rückläufig zu sein, was auf den sehr großzügigen Einsatz von Doxycyclin bei Zervizitis und Urethritis zurückgeführt wird.

Sehr häufig ist die Infektion klinisch aber asymptomatisch. Ein jährliches Screening bei sexuell aktiven Frauen im Alter von 
Tab. 3 Therapieempfehlungen bei einer Chlamydieninfektion

\begin{tabular}{ll} 
Deutsche STD-Gesellschaft 2001 & WHO Guidelines 2001 \\
Azithromycin $1 \mathrm{~g}$ po einmalig & Azithromycin $1 \mathrm{~g}$ po einmalig \\
Doxycyclin $2 \times 100 \mathrm{mg} /$ die 7 Tage & Doxycyclin $2 \times 100 \mathrm{mg} /$ die 7 Tage \\
\hline Alternativ & Alternativ \\
Erythromycin $4 \times 500 \mathrm{mg} /$ die 7 Tage & Erythromycin $4 \times 500 \mathrm{mg} / \mathrm{d} 7$ Tage \\
Erythromycin $2 \times 500 \mathrm{mg} /$ die 14 Tage & Roxithromycin $2 \times 150 \mathrm{mg} / \mathrm{d} 7$ Tage \\
Tetrazyklin $4 \times 500 \mathrm{mg} /$ die 7 Tage & Clarithromycin $2 \times 250 \mathrm{mg} / \mathrm{d} 7$ Tage \\
Ofloxacin $2 \times 300 \mathrm{mg} /$ die 7 Tage & Ofloxacin $2 \times 200 \mathrm{mg} /$ die 7 Tage
\end{tabular}

\section{CDC Guidelines 2006}

Azithromycin $1 \mathrm{~g}$ po einmalig

Doxycyclin $2 \times 100 \mathrm{mg} / \mathrm{die} 7$ Tage

Alternativ

Erythromycin $4 \times 500 \mathrm{mg} /$ die 7 Tage

Ofloxacin $2 \times 300 \mathrm{mg} /$ die 7 Tage

Levofloxacin $500 \mathrm{mg} /$ die 7 Tage
20 - 25 Jahren und bei symptomatischen Patienten wird deshalb empfohlen. Mit dem PCR Nachweis steht eine Methode mit hoher Sensitivität und Spezifität zur Verfügung.

Die Therapie einer Chlamydieninfektion ist durch Studien ausreichend abgesichert, was zu übereinstimmenden Therapieempfehlungen nahezu aller Fachgesellschaften geführt hat (s. - Tab. 3).

Die Sanierungsraten von 97-98\% einer Einzeittherapie mit Azithromycin $1 \mathrm{~g}$ oder die Wochentherapie mit Doxycyclin $200 \mathrm{mg} /$ Tag sind gleich. Die Patienten sollten aber bei einer Einzeittherapie mit Azithromycin zu einer sexuellen Abstinenz von 1 Woche und bei Doxycyclin bis zum Ende der Therapie angehalten werden. Zur Vermeidung einer Reinfektion sind die Sexualpartner zu untersuchen und zu therapieren, falls ein sexueller Kontakt in den vorausgegangenen 60 Tagen stattgefunden hat oder entsprechende Beschwerden angegeben werden. Eine mögliche Doppelinfektion mit Gonokokken ist bei den Untersuchungen zu berücksichtigen.

Nach erfolgter Therapie wird eine Nachuntersuchung für nicht erforderlich gehalten, falls die Beschwerden rückläufig sind. Eine solche sollte mit einem Mindestabstand von 3 Wochen zur Behandlung stattfinden, um falsch-negative Kultur-Ergebnisse bei zu geringer Keimzahl oder falsch-positive PCR-Ergebnisse bei verbliebenen avitalen Keime zu vermeiden. Rezidive beruhen nicht auf einer Antibiotikaresistenz der Chlamydien, sondern auf einer Incompliance bei der Primärtherapie oder auf einer Reinfektion bei ineffektiver Partnersanierung.

\section{Herpes genitalis \\ $\nabla$}

Der Herpes genitalis ist die zahlenmäßig häufigste STD. Das klinische Bild von schmerzhaften genitalen oder glutealen Bläschen und Ulzerationen ist nicht sehr spezifisch. Bei Auslösung durch Herpesviren kommen sowohl HSV1 als auch HSV2 als Pathogene in Frage. Die Prävalenzzahlen sind so hoch, weil die Infektion lebenslang besteht. Die Wahrscheinlichkeit der Ausscheidung von infektiösen Viruspartikeln, auch bei asymptomatischen Personen, scheint aber bei HSV2 höher zu sein. Die Herpesdiagnostik einschließlich Typisierung sind durch eine Viruskultur und Immunofluoreszenzuntersuchungen möglich, die Sensitivität der Methoden ist aber nicht optimal. Die alternativ mögliche PCR Diagnostik ist jedoch in Deutschland im GKV Bereich nicht mehr erstattungsfähig. Serologische Untersuchungen bei Verdacht auf Herpesinfektionen sind nur bei der Primoinfektion aussagekräftig. Die Sensitivität des serologischen HSV2 Nachweises liegt bei 80-98\%, wobei aber bei initialen Infektionen falsch-negative Befunde auftreten können.

Die Therapie ist davon abhängig, ob die eine Primoinfektion oder ein Rezidiv vorliegt. Die Therapieempfehlungen der führenden Institutionen sind nahezu deckungsgleich (s. $\bullet$ Tab. 4).

Die genannten Substanzen sind für die Indikation Herpes genitalis zugelassen. Eine Therapie im akuten Stadium beseitigt weder eine latente Virusinfektion noch beeinflusst sie das Risiko, die Frequenz und die Schwere von Rezidiven. Eine topische Therapie mit antiviralen Substanzen bietet nur einen minimalen klinischen Benefit, die Anwendung von entsprechenden Substanzen wird deshalb in der CDC Leitlinie nicht empfohlen.

Tab. 4 Therapieempfehlungen für Herpes genitalis

Deutsche STD-Gesellschaft 2001
Primoinfektion
Aciclovir $3 \times 400 \mathrm{mg} / \mathrm{d} 7$ - 10 Tage
Aciclovir $5 \times 200 \mathrm{mg} / \mathrm{d} 7$ - 10 Tage
Famciclovir $3 \times 250 \mathrm{mg} / \mathrm{d} 7$ - 10 Tage
Valaciclovir $2 \times 1 \mathrm{~g} / \mathrm{d} 7$ - 10 Tage
Herpes rezidivans
Aciclovir $3 \times 400 \mathrm{mg} / \mathrm{d} 5$ Tage
Aciclovir $5 \times 200 \mathrm{mg} / \mathrm{d} 5$ Tage
Famciclovir $2 \times 125 \mathrm{mg} / \mathrm{d} 5$ Tage
Valaciclovir $2 \times 500 \mathrm{mg} / \mathrm{d} 3$ - 5 Tage
Suppressive Dauertherapie bei Rezidiven
mind. alle 6 Wochen
Aciclovir $3 \times 400 \mathrm{mg} / \mathrm{d}$
Famciclovir $2 \times 250 \mathrm{mg} / \mathrm{d}$
Valaciclovir $1 \times 500 \mathrm{mg} / \mathrm{d}$

\section{IUSTI/WHO Guidelines 2001}

Primoinfektion

Aciclovir $5 \times 200 \mathrm{mg} / \mathrm{d} 5$ Tage

Famciclovir $3 \times 250 \mathrm{mg} / \mathrm{d} 5$ Tage

Valaciclovir $2 \times 1 \mathrm{~g} / \mathrm{d} 5$ Tage

\section{Herpes rezidivans}

Aciclovir $5 \times 200 \mathrm{mg} / \mathrm{d} 5$ Tage

Famciclovir $2 \times 125 \mathrm{mg} / \mathrm{d} 5$ Tage

Valaciclovir $2 \times 500 \mathrm{mg} / \mathrm{d} 5$ Tage

Suppressive Dauertherapie bei $>6$ Rezidiven / Jahr

Aciclovir $2 \times 400 \mathrm{mg} / \mathrm{d}(4 \times 200 \mathrm{mg} / \mathrm{d})$

Famciclovir $2 \times 250 \mathrm{mg} / \mathrm{d}$

Valaciclovir $1 \times 500-1000 \mathrm{mg} / \mathrm{d}$

\section{CDC Guidelines 2006 \\ Primoinfektion \\ Aciclovir $3 \times 400 \mathrm{mg} / \mathrm{d} 7$ - 10 Tage \\ Aciclovir $5 \times 200 \mathrm{mg} / \mathrm{d} 7-10$ Tage \\ Famciclovir $3 \times 250 \mathrm{mg} / \mathrm{d} 7$ - 10 Tage \\ Valaciclovir $2 \times 1 \mathrm{~g} / \mathrm{d} 7-10$ Tage}

\author{
Herpes rezidivans \\ Aciclovir $3 \times 400 \mathrm{mg} / \mathrm{d} 5$ Tage \\ Aciclovir $2 \times 800 \mathrm{mg} / \mathrm{d} 5$ Tage \\ Aciclovir $3 \times 800 \mathrm{mg} / \mathrm{d} 2$ Tage \\ Famciclovir $26 \times 125 \mathrm{mg} / \mathrm{d} 5$ Tage \\ Valaciclovir $2 \times 500 \mathrm{mg} / \mathrm{d} 3$ Tage \\ Valaciclovir $1 \mathrm{~g} / \mathrm{d} 5$ Tage \\ Suppressive Dauertherapie bei \\ $>6$ Rezidiven / Jahr \\ Aciclovir $2 \times 400 \mathrm{mg} / \mathrm{d}$ \\ Famciclovir $2 \times 250 \mathrm{mg} / \mathrm{d}$ \\ Valaciclovir $1 \times 500-1000 \mathrm{mg} / \mathrm{d}$
}


Tab. 5 Therapieempfehlungen für Condylomata acuminata-Infektionen

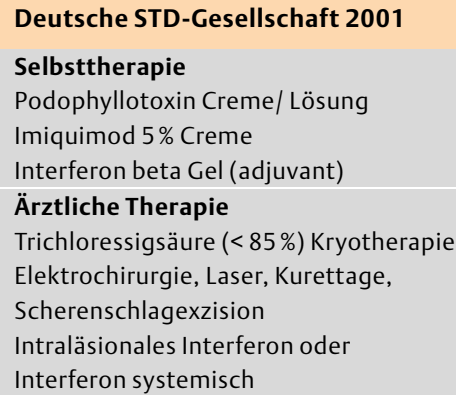

\section{IUSTI/WHO Guidelines 2001}

Selbsttherapie

Podophyllotoxin Creme/ Lösung Imiquimod 5\% Creme

\section{Ärztliche Therapie}

Kryotherapie

Trichloressigsäure $80-90 \%$

Chirurgische Verfahren (Exzision,

Curettage, Kauterisation)
CDC Guidelines 2006

Selbsttherapie

Podophyllotoxin Gel oder Lösung

Imiquimod 5\% Creme

\section{Ärztliche Therapie}

Kryotherapie

Podophyllin 10-25\%

Trichloressigsäure 80 - $90 \%$

Chirurgische Verfahren (Exzision, Curettage, Kauterisation)

Alternativ

Intraläsionales Interferon

Laserchirurgie

\section{Deutsche STD Gesellschaft 200}

$0,5 \%$ Permethrinlösung 30Minuten Pyrethrine 10-30 Minuten

\section{WHO Guidelines 2001}

$1 \%$ Permethrincreme 10 Minuten Pyrethrine 10 Minuten

\section{CDC Guidelines 2002}

$1 \%$ Permethrincreme 10 Minuten Pyrethrine 10 Minuten
Tab. 6 Therapieempfeh-

lungen bei Filzlausbefall
Die Indikationsstellung einer langfristigen ( $>6-12$ Monate) kontinuierlichen Prophylaxe wird von der Häufigkeit der Rezidive und der Stärke der jeweiligen Beschwerden abhängig sein. Die CDC geht davon aus, dass jährlich mindestens 6 Herpesschübe eine Dauerprophylaxe rechtfertigen. Die nicht unerheblichen Kosten einer suppressiven Dauertherapie werden sicherlich auch ein Kriterium für die Wahl des entsprechenden Medikamentes sein. Eine Dauertherapie reduziert die Zahl der Schübe um 70-80\%. Die betroffenen Patienten scheiden auch im asymptomatischen Intervall Herpesviren aus. Es ist bislang aber nicht bekannt, ob der unter einer Langzeitprophylaxe verringerten Virusausscheidung eine klinische Relevanz bezüglich der Infektiosität zukommt.

\section{Genitalulcera - Ulcus molle}

Der Nachweis von akut aufgetretenen Genitalulzerationen erfordert den Ausschluss einer STD, insbesondere der Lues, einer Herpesinfektion und des Ulcus molle. Zahlenmäßig häufiger sind natürlich beim Dermatologen Patienten mit nicht infektiösen Erkrankungen wie dem M. Behçet, Arzneiexanthemen und toxischer Dermatitis oder bakterieller/mykotischer Balanitis und Vulvitis. Diagnostik und Therapie der Lues und der Herpesinfektion sind in den entsprechenden Abschnitten besprochen worden. Das Ulcus molle kommt in Afrika (Gambia, Kenia), in der Karibik und in Südwestasien häufig vor. In den USA und in Osteuropa ist es nicht selten, in Deutschland gibt es aber nur eine sehr kleine Zahl von Dermatologen, die eine signifikante Fallzahl gesehen haben. Dies mag sicher auch am schwierigen Nachweis des Erregers liegen. Der kulturelle Nachweis erfordert Spezialmedien, der Direktnachweis von „fischzugartig angeordneten Bakterien“ in der Gramfärbung setzt die Kenntnis des entsprechenden Bildes voraus. Beim Auftreten eines Patienten mit der Fragestellung sind beide Bedingungen wahrscheinlich nicht erfüllt. Der klinische Befund ist durch das Vorliegen eines dolenten Genitalulcus und einer dolenten Lymphadenopathie gekennzeichnet. Die Therapie (nach CDC und IUSTI) besteht in der einmaligen Gabe von $1 \mathrm{~g}$ Azithromycin po oder $250 \mathrm{mg}$ Ceftriaxon im. Auch wenn Resistenzen beobachtet wurden, können alterna- tiv Ciprofloxazin $2 \times 500 \mathrm{mg} / \mathrm{d}$ für 3 Tage oder Erythromycin $3 \times 500 \mathrm{mg} / \mathrm{d}$ für 7 Tage eingesetzt werden.

\section{Condylomata acuminata}

Feigwarzeninfektionen sind im jüngeren Erwachsenenalter sehr häufig. Inapperente HPV Virusinfektionen scheinen bei der Mehrzahl der Erwachsenen zu bestehen. In den USA wurde kürzlich bei 44\% der Frauen von 20-24 Jahren eine Besiedelung des Genitalbereichs mit HPV Viren nachgewiesen [9].

Die therapeutische Zielsetzung bei Feigwarzen hat sich in den letzten Jahren gewandelt. Es soll durch die Behandlung nur versucht werden, eine Beseitigung der Symptome zu erreichen. Eine Eradikation der Virusinfektion ist wohl nicht möglich. Es ist darüber hinaus aber nicht geklärt, ob sich durch eine therapeutische Reduktion der Viruslast die Infektiosität ändert. Nach den Angaben der CDC Leitlinien aus dem Jahr 2006 gibt es bislang keinen Beweis dafür, dass die Präsenz von genitalen Warzen oder deren Therapie mit der Entstehung des Zervixkarzinoms assoziiert ist.

Die Behandlungsmodalitäten von Genitalwarzen sollten die Wünsche des Patienten, die vorhandenen Möglichkeiten und die Erfahrung des Therapeuten berücksichtigen (s. Tab. 5). Es gibt bislang keine definitiven Beweise für die Überlegenheit einer der vorgeschlagenen Therapieformen.

Bei der Wahl der Therapieform ist darauf zu achten, dass der Behandler mit der gewählten Behandlung sehr gut vertraut ist. Podophyllin ist eine seit Jahrzehnten angewendete Substanz, deren Effektivität unzweifelhaft ist. Auch bei erfahrenen Behandlern können dadurch (heftige) Nebenwirkungen auftreten. Da mit Podophyllotoxin eine weniger toxische Substanz sogar als Fertigarzneimittel verfügbar ist, sollte wirklich sehr gut überlegt werden, ob man an dem alten Podophyllin festhalten will. Auch Imiquimod-Creme kann bei entsprechend disponierten Personen zu massiven lokalen und systemischen Reaktionen führen, weshalb bei der erstmaligen Anwendung nur ein kleines Areal behandelt werden sollte.

Die klinische Diagnosestellung wird nach den CDC Leitlinien für ausreichend erachtet. Es liegen darüber hinaus keine Daten vor, die für die Notwendigkeit einer Bestimmung des HPV-Subtyps 
in der Routinediagnostik sprechen. Untersuchungen in Hochrisikokollektiven haben gezeigt, dass Mehrfachinfektionen mit verschiedenen HPV Typen häufig sind.

Der Nachweis subklinischer Feigwarzen ist durch den Essigsäuretest möglich (3-5\% Essigsäure einige Minuten im Tupfer auftragen, suspekte Areale färben sich weiß). Bislang ist nicht bekannt, ob solche subklinischen Läsionen weniger infektiös sind als exophytische Feigwarzen. Eine Behandlung subklinischer Läsionen wird aber nicht für erforderlich gehalten. Konsequenterweise ist nach den CDC Leitlinien eine außerplanmäßige Partneruntersuchung nicht erforderlich, wenn regelmäßige zytologische Zervixabstriche im Rahmen der Krebsvorsorge erfolgen. Nach erfolgter Therapie werden Folgeuntersuchungen in 3-monatigen Abständen empfohlen.

Bei immundefizienten Patienten (HIV, transplantierte Patienten) sind die angeführten Therapieformen wirksam, es muss aber mit vermehrten Rezidiven gerechnet werden. Da die Inzidenz von Plattenepithelkarzinomen erhöht ist, welche in Feigwarzen entstehen oder solche imitieren, sollte eine histologische Untersuchung der Läsionen erfolgen. Dies gilt insbesondere für das Analkarzinom bei analen Feigwarzen. Die CDC hält aktuell die Datenlage für noch nicht ausreichend, ein generelles zytologisches Screening (analog zum Zervikalabstrich) auf Analkarzinome bei homosexuellen HIV Patienten zu empfehlen.

Kürzlich wurde ein Impfstoff gegen kanzerogene Papillomviren zugelassen. Zur Zeit werden Studien mit der Fragestellung durchgeführt, ob eine Impfung auch die Inzidenz von Analkarzinomen reduzieren und ob eine Impfung nach (!) der Erstdiagnose von Condylomen die Anzahl der klinischen Rezidive (ausgelöst möglicherweise durch andere HPV Typen) reduzieren kann.

\section{Filzläuse (Phthiriasis)}

$\nabla$

Der Befall mit Filzläusen sollte als Marker für das gleichzeitige Bestehen einer STD gewertet werden. Die Diagnosestellung ist prima vista möglich. Therapieversager beruhen meist auf inkompletter Therapie oder Reinfektionen. Eine Partnerbehandlung ist deshalb erforderlich, ebenso wie eine Sanierung des häuslichen Umfeldes und der Kleidung. Die Therapieempfehlungen weltweit sind jetzt übereinstimmend, nachdem Lindan nicht mehr erhältlich ist (s. Tab. 6). Interessanterweise findet sich in keiner Therapieleitlinie der Hinweis, dass auch die Entfernung der Körperhaare den Menschen für die Filzläuse unwohnlich macht.

Filzläuse können auch die Wimpern besiedeln. Eine oklusive Behandlung mit Augenvaseline $2 \times$ täglich über 10 Tage soll zum Erstickungstod der Läuse führen, alternativ kommt die Anwendung (10 Minuten) von $1 \%$ Permethrinlösung bei geschlossenen Augen in Betracht. Die Wertigkeit von Pilocarpin (Pilogel) ist nicht beschrieben.

Die zunehmende Internationalisierung, die de facto eine Amerikanisierung ist, verleiht den CDC Empfehlungen zur Diagnostik und Therapie der sexuell übertragbaren Erkrankungen eine gewisse Leitfunktion. Die Institution wird diesem Anspruch zu- sätzlich dadurch gerecht, indem sie regelmäßige Fortschreibungen veröffentlicht. Die europäischen Regionalbüros der IUSTI und der WHO veröffentlichen wichtige Daten zu epidemiologischen Trends der STD, die Therapieempfehlungen erfolgen aber in größeren Abständen. Bedingt durch die Globalisierung und den zunehmenden Reiseverkehr sind die Unterschiede zu den CDC Empfehlungen aber nur noch marginal.

Aktuelle Therapie-Leitlinien zeigen den allgemein akzeptierten diagnostischen und therapeutischen Spielraum auf. Sie sollten in unserem Praxisschreibtisch liegen und auch eingesehen und umgesetzt werden, wenn ein entsprechender Patient vorstellig wird.

Wenn die Dermatologen sich weiterhin intensiv mit der Diagnostik und Therapie der sexuell übertragenen Krankheiten befassen, werden andere Fachgebiete unsere zentrale medizinische Kompetenz für die betroffenen Patienten anerkennen. Nur so kann zukünftig diese „Wurzel“ unseres Fachgebietes als Teilgebiet der Dermatologie erhalten werden.

\section{Abstract}

\section{Sexually Transmitted Diseases: New Treatment Guidelines Valuable Recommendations for Daily Practice $\nabla$}

In recent years medical societies and health organizations have published new treatment guidelines for sexually transmitted diaeses (STD). A significant increase in STD incidence globally, rapidly spreading antimicrobial resistances and introduction of new antibiotics led to frequent updates. Noteworthy, differences in treatment recommendations turned marginal, and were mostly due to publication date. This review focuses on recent epidemiologic trends and new treatment recommendations, which are significant for daily practice of dermatovenerology.

\section{Literatur}

1 Centers for Disease Control and Prevention. Sexually transmitted diseases Treatment guidelines 2006. MMWR 55 (No. RR-11), www.cdc.gov/std/treatment//2006/toc.htm

2 Centers for Disease control and Prevention. Sexually tranmitted Disease Surveillance 2006. Supplement. www.cdc.gov/STD/Syphilis2006/ SyphilisShort.pdf

3 IUSTI/WHO. European STD Guidelines. www.iusti.org/sti-information/ pdf/guidelines.pdf

4 IUSTI - European Guidelines on the Management of Syphilis 2007 (Draft). www.iusti.org/sti/european_guidelines.pdf

5 Leitlinie der Deutschen STD-Gesellschaft: Diagnostik und Therapie der Syphilis 2005. www.awmf-leitlinien.de (Leitlinie 059/002)

6 Robert Koch Institut. Syphilis(Lues). RKI-Ratgeber/Merkblätter für Ärzte. www.rki.de

7 WHO. Trend in sexually transmitted infections and HIV in the European region, 1980-2005. www.euro.who.int/document/RC56/gtb01b. pdf

8 Gille G, Klapp C, Diedrich K et al. Chlamydien - eine heimliche Epidemie unter Jugendlichen - Prävalenzbeobachtungen bei jungen Mädchen in Berlin. Dtsch Aerzteblatt 2005; 102: A2021

9 Dunne EF, Unger ER, Sternberg $M$ et al. Prevalence of HPV infection among females in the United States. JAMA 2007; 297: 813-819 